\title{
long-term performance
}

2. Department of Biology, Faculty of Science, University of Southern Denmark, 5230 Odense, Denmark.

3. Molecular Invertebrate Systematics and Ecology Laboratory, Graduate School of Engineering and Science, University of the Ryukyus, Nishihara, Okinawa, Japan.

4. Tropical Biosphere Research Centre, University of the Ryukyus, Nishihara, Okinawa, Japan.

5. School of Life Sciences, University of Technology Sydney, Ultimo, NSW 2007, Australia

6. School of Life and Environmental Science, The University of Sydney, Camperdown, NSW 2006, Australia.

7. Australian Research Council Centre of Excellence for Coral Reef Studies, School of Biological Sciences, The University of Queensland, Brisbane, QLD 4072, Australia.

8. Kuroshio Biological Research Foundation, Nishidomari, Otsuki-cho, Kochi, Japan.

9. Solitary Islands Marine Park, Department of Primary Industries, Coffs Harbour, NSW 2450, Australia.

10. Department of Zoology, University of Oxford, Oxford, 11a Mansfield Rd, OX1 3SZ United Kingdom.

11. Centre for Biodiversity and Conservation Science, School of Biological Sciences, University of Queensland, Brisbane, QLD, 4072 Australia.

12. Max Planck Institute for Demographic Research, Konrad Zuße Straße 1, 18057 Rostock, Germany.

${ }^{* * *}$ Share equal contribution

"Corresponding authors: MB collected field data, with JC, CF, MBr analysing the data. JC, RS-G, and MB led the writing of the manuscript with all authors contributing critically to the writing and giving final approval for publication. dynamics. 


\section{$31 \quad$ Abstract}

The current exposure of species assemblages to high environmental variability may grant them resilience to future increases in climatic variability. In globally threatened coral reef ecosystems, management seeks to protect resilient reefs within variable environments. Yet, our lack of understanding for the determinants of coral population performance within variable environments hinders forecasting the future reassembly of coral communities. Here, using Integral Projection Models, we compare the short- (i.e., transient) and long-term (i.e., asymptotic) demographic characteristics of tropical and subtropical coral assemblages to evaluate how thermal variability influences the structural composition of coral communities over time. Exploring spatial variation across the dynamics of functionally different competitive, stress-tolerant, and weedy coral assemblages in Australia and Japan, we show that coral assemblages trade-off long-term performance for transient potential in response to thermal variability. We illustrate how coral assemblages can reduce their susceptibility towards environmental variation by exploiting volatile short-term demographic strategies, thus enhancing their persistence within variable environments. However, we also reveal considerable variation across the vulnerability of competitive, stress-tolerant, and weedy coral assemblages towards future increases in thermal variability. In particular, stress-tolerant and weedy corals possess an enhanced capacity for elevating their transient potential in response to environmental variability. Accordingly, despite their current exposure to high thermal variability, future climatic shifts threaten the structural complexity of coral assemblages, derived mostly from competitive coral taxa within highly variable subtropical environments, emulating the degradation expected across global coral communities. 


\section{Introduction}

Coral reefs worldwide are threatened by unprecedented climatic and anthropogenic disruption

$(1,2)$, with their conservation now reliant on enhancing the resilience of coral communities, specifically their capacity to resist and recover from increased climatic variability $(3,4)$.

Changes in environmental regimes provoke spatial shifts in the performance and distribution of populations, which ultimately upscale to the compositional reassembly of biological communities $(5,6)$. Exposure to increased environmental variability is, however, expected to indirectly augment the capacity for populations to resist and recover from repeated disturbances (7, 8). Yet, nuanced relationships between population characteristics and biophysical conditions ensure inconsistent responses towards climate shifts, even across populations within the same region $(9,10)$. For instance, differential population sensitivities to habitat change can accelerate or reverse expected poleward range shifts in response to climate warming (10). Thus, anticipating the future resilience of natural communities requires understanding the collective vulnerabilities of their constituent populations (11) and the determinants underpinning their resilience to increasingly recurrent disturbances $(12,13)$. However, regional variations in the response of coral assemblages to climatic disturbances have generated misconceptions in our interpretation of the status of coral communities worldwide, with global population sizes of many species perhaps larger than previously anticipated (14). Only by linking the mechanisms underpinning heterospecific variation across the responses of populations to environmental variability can one predict the resilience of biological communities to increased climatic variability $(11,15,16)$. Evidently, to accurately forecast the ongoing reassembly of global coral communities we must improve our understanding for how environmental variability shapes coral population performance across community- and regional-scales (7).

To explore the performance of populations exposed to recurrent disturbances within variable environments, their transient (i.e., short-term) dynamics must be considered (17-20). 
80

Yet, asymptotic (i.e., long-term) population growth rate $(\lambda)$, a metric that describes temporal changes in population size at stationary equilibrium (21), is the predominant metric used to quantify population performance $(21,22)$. In reality, though, natural populations are exposed to repeated disturbances that favour the persistence of transient conditions, preventing the emergence of stationary equilibria $(17,20,23)$. Within variable environments, repeated disturbances impose short-term changes upon the structure of populations that can elevate (amplify) or diminish (attenuate) their growth rates, resulting in population performance characteristics deviating from long-term expectations $(18,24)$. Quantifying how transient population performance deviates from long-term expectations (henceforth transient potential) is therefore crucial for predicting the success or failure of natural populations (25); an approach that remains neglected within coral research (19).

Located at the intersection of tropical and temperate ecoregions, subtropical coral communities provide an opportunity for evaluating the abiotic determinants of coral population performance (26-28). Over recent decades, subtropical coral communities have undergone transformation with various coral taxa undergoing poleward range expansions in response to shifting thermal regimes (29-33). However, at higher latitudes, coral communities are exposed to enhanced seasonality and cooler winter temperatures, and thus experience greater abiotic variability relative to their tropical reef counterparts (34). Over time, the persistence of populations within stable environments diminishes their capacity for tolerating novel environmental states, thus reducing their resilience towards future climatic shifts (8). Consequently, the endurance of subtropical coral communities within variable high-latitude environments is expected to enhance their abiotic resilience (7). Indeed, as seen across other communities (e.g., plants; 35), enhanced transient potential can elevate the performance of acroporid coral populations within variable subtropical environments (36). Yet, without knowledge for how differing coral assemblages utilise demographic strategies to mediate their 
performance in response to environmental variability, we will be unable to forecast the impacts of increasing climatic variability on the condition of global coral reef ecosystems.

In species rich communities, evaluating ecological dynamics requires a trait-based approach to condense vast quantities of demographic detail (37). Given the diversity of coral reef ecosystems, exploring patterns across the demographic characteristics of co-occurring coral species presents a logistical challenge (38). Yet, this is a challenge that can be navigated by pooling individuals based on shared trait characteristics. Morphological, physiological and phenological functional traits influence the fitness of individuals and thus determine the demographic characteristics of their populations (39), their responses to disturbances (40), and subsequently the assembly of biological communities (41-43). Indeed, functional trait characteristics impact upon the demographic properties of coral populations (e.g., colony growth and reproduction; 44, 45), mediating their ability to respond to local abiotic patterns (46). Given such strong links between coral traits and demographic performance, the categorisation of coral taxa into competitive, stress tolerant, generalist and weedy life history assemblages (sensu 47) is used to evaluate broadscale patterns in coral community reassembly (48-50). The trait-based assessment of coral community assembly also offers greater insight into the wider implications of ongoing community shifts than taxonomic-based assessments, thereby aiding the management of coral reef ecosystems (50).

Here, we investigate how the performance characteristics of tropical and subtropical coral populations map onto patterns of thermal variability across assemblages of competitive, stress-tolerant, and weedy coral taxa. Using Integral Projection Models (IPMs; 51), we quantify the association between different dimensions of thermal variability (monthly mean sea surface temperature [SST], monthly SST variance, and monthly SST frequency spectrum) and the transient potential and long-term performance characteristics of tropical and subtropical coral assemblages in southern Japan and eastern Australia (Fig. 1). Specifically, we anticipate that, 
in accordance with their exposure to elevated thermal variability, subtropical coral assemblages will possess greater transient potential in comparison to tropical assemblages. We expect that this pattern will persist irrespective of functional strategy, corresponding with the need for subtropical coral populations to exploit periodically disturbed environments.

\section{Results and Discussion}

Our analyses reveal a trade-off between long-term performance and transient potential across the examined coral assemblages. We find that this trade-off corresponds with the exposure of coral populations to thermal variability along a gradient from warmer, more stable environments to cooler, more variable conditions (Fig. 2). Using partial least squares regression, we evaluated how patterns in the long-term performance, demographic recovery, and transient potential, of coral populations conform with their exposure to abiotic variability. We obtained estimates of long-term population performance (asymptotic population growth rate, $\lambda$ ), demographic recovery (damping ratio $[\rho]$, i.e., a relative measure of the time needed for a population to converge to a stable equilibrium; 21), and transient potential (transient envelope $[T E]$, i.e., the difference between maximum and minimum population size following disturbance; 24, 52) from IPMs depicting the dynamics of tropical and subtropical assemblages of competitive, stress-tolerant, and weedy coral taxa in Japan and Australia (Fig. 1; Supplementary S1 \& S2). Furthermore, we quantified the exposure of these assemblages to thermal variability using three measures of local SST regimes: monthly mean SST $\left(\bar{x}_{s s t}\right)$, monthly SST variance $\left(c v_{s s t}\right)$, and monthly SST frequency spectrum $\left(\beta_{s s t}\right.$; Supplementary S3).

Notably, the coral assemblages exposed to more variable thermal conditions display enhanced transient potential. Our partial least squares regression explains $92.17 \%$ of the variance in the three measures of thermal exposure $\left(\bar{x}_{s s t}, c v_{s s t}\right.$, and $\left.\beta_{s s t}\right)$, whilst also capturing $37.43 \%$ of the variance in long-term performance $(\lambda)$, demographic recovery $(\rho)$, and transient 
154

potential (TE; Fig. 2, $\left.r_{[y]}^{2}\right)$. The first partial least squares regression component reflects a gradient in SST variability, capturing $60.97 \%$ of the variance in thermal conditions experienced by our examined coral assemblages. It is along this component that divergent patterns within estimates of $\lambda$ and $T E$ are most pronounced. Subsequently, estimates of $T E$ are positively correlated with the measures of thermal variability $\left(c v_{s s t}\right)$ and frequency spectrum $\left(\beta_{s s t}\right)$, whilst higher $\lambda$ estimates associate with warmer mean monthly SSTs ( $\bar{x}_{s s t}$; Fig. 2). Meanwhile, damping ratio $(\rho)$ estimates are aligned with the second partial least squares regression component describing secondary patterns in the mean $\operatorname{SST}\left(\bar{x}_{s s t}\right)$ and frequency $\left(\beta_{s s t}\right)$ variables. Enhanced transient potential is thought to buffer the performance of populations in response to elevated abiotic variability, thereby underpinning their capacity to exploit more variable environments $(35,53)$. However, variation in transient potential across the assemblages of differing coral taxa, in response to increased thermal variability (Fig. 3), suggests that exposure to abiotic variability alone does not assure resilience towards future climatic variability.

The trade-off between long-term performance and transient potential does not manifest consistently across the examined tropical and subtropical coral assemblages (Fig. 3A \& Table 1), with inter-specific variation mediated instead by characteristics of population turnover (Fig. 3B \& C). We explore inter-assemblage variation across estimates of long-term performance and transient population growth potential. Again, we quantified long-term performance using $\lambda$, whilst the demographic stability index $(D S I)$ calculated from our IPMs provided a measure of transient growth potential. A three-way ANOVA reveals significant interactions between the three factors of assemblage classification (competitive, stress-Tolerant or weedy), ecoregion (tropical vs. subtropical), and country (Australia vs. Japan; ANOVA $\lambda$ : $\mathrm{F}_{2,11562}=$ 5698.47, $p<0.001$; ANOVADSI: $\left.\mathrm{F}_{2,11581}=589.8, p<0.001\right)$. Despite this, the tropical assemblages routinely possess higher estimates of $\lambda$ relative to their corresponding subtropical counterparts (Tukey: $p<0.001$ in all cases; Table 1). The one exception were weedy corals in 
Japan, where $\lambda$ is highest in the subtropics $\left(\lambda_{\mathrm{t}]}=0.760\right.$ [95\% CI: $\left.0.750,0.770\right], \lambda_{[\mathrm{s}]}=0.807$ $[0.802,0.812] ; p<0.001)$. Alternatively, the subtropical coral assemblages typically possess a greater capacity for amplifying population growth following a disturbance than the tropical assemblages (Fig. 3A). However, this pattern is not consistent across life history strategies, with competitive assemblages exhibiting the opposite trend in Australia $(p<0.001)$, and no variation in Japan $(p=0.999)$.

The long-term performance and transient potential of the coral assemblages corresponds with patterns in their generation time (Fig. 3B \& C). To further evaluate the drivers mediating coral population performance within variable environments we used Type 2 linear regression (54) to explore the relationship between estimates of generation time (T, i.e., the time needed for individuals of a population to be replaced; 55), long-term performance $(\lambda)$, and transient potential (TE) calculated from our IPMs. Generation time is a strong predictor of longterm population growth rate $\left(r^{2}=0.704\right)$, with long-term performance increasing with generation time (Fig. 3B). Conversely, longer generation times are associated with reduced transient potential (Fig. 3C; $r^{2}=0.409$ ). Hence, our observed trade-off between long-term performance and transient potential, in response to thermal variability, manifests inconsistently across our examined tropical and subtropical coral assemblages, due to variation in their characteristics of temporal population turnover.

\section{Transient buffering in variable environments}

Principally, a trade-off between long-term performance and transient potential implies that long-term performance does not predict the capacity for populations to endure repeated disturbances. Simultaneously, however, it also suggests that whilst enhanced transient potential may enable natural populations to persist within variable environments, it comes at a cost to their long-term performance. Historically, variability in population growth rate was thought to 
diminish individual fitness (56), thus hindering the persistence of populations (57). This understanding formed the basis of the demographic buffering hypothesis, whereby populations can minimise the influence of environmental variability on their long-term performance by limiting temporal variability in crucial vital rates (e.g., survival, development and reproduction; 58). Thus, variable environments were assumed to select for populations with the ability to buffer key vital rates, thereby reducing temporal variation in performance characteristics $(56$, 58, 59). More recently, however, enhanced transient potential has been presented as an adaptive mechanism that allows populations to exploit regions with high environmental variability (35). Indeed, Ellis \& Crone (53) demonstrated how increased transient potential can buffer the effects of stochastic conditions on population growth rates, an effect that was increasingly evident in populations possessing lower $\lambda$ estimates. Thus, it is not unexpected, that coral assemblages established within variable environments, would possess enhanced transient potential (Fig. 2). Yet, if the vital rate schedules of these assemblages have indeed evolved specifically to maximise their short-term performance, this would likely carry an energetic cost to their long-term performance characteristics.

Our finding that transient potential is greatest in coral assemblages displaying reduced long-term performance contrasts with previous work on mammals and plants showing a positive association between population growth rates and transient potential (e.g., 60, 61). Faster population growth rates are assumed of populations characterised by faster individual development and high fecundity (62), with these populations also expected to exhibit greater variability in size following disturbances (61). Whilst it is concerning that each of our surveyed assemblages are in, or close to, a state of long-term decline $(\lambda<1$; Table 1$)$, projected longterm performance is greatest in the tropical assemblages which also typically display lower transient amplification in population growth (Fig. 3A). These findings agree with evidence that transient potential is mediated by population turnover. Populations exhibiting longer 
generation times typically display reduced temporal variability in size corresponding with the fact that higher individual survival reduces the need to counteract disturbances (60); a pattern that we show to be evident in our examined coral assemblages (Fig. 3C). Accordingly, our observation that long-term performance is positively correlated with generation time in coral populations (Fig. 3B), perhaps presents a more intriguing result. Although, with the long- and short-term performance characteristics of coral assemblages corresponding with their relative exposure to thermal variability (Fig. 2), this finding further implies that trading off long-term performance for transient potential is an adaptive characteristic in coral populations.

Forecasting community reassembly

237 Here we show that stress-tolerant and weedy coral taxa possess more pronounced transient amplification, highlighting a potential mechanism supporting their persistence at higher latitudes. Using a subset of the dataset presented here, Cant et al. (36) recently demonstrated how short-term increases in population growth following disturbance could support the establishment of a subtropical coral assemblage of competitive Acropora spp. in southern Japan. However, subtropical-tropical variation in the amplification capacity of competitive coral assemblages appears minimal in comparison to the variation we observe across the stresstolerant and weedy assemblages in both Australia and Japan (Fig. 3A). Weedy corals comprise species that exhibit small colony sizes, fast growth rates, and internal fertilisation, producing larvae that settle quickly after release $(47,63)$. Together, these strategies support faster population turnover, enabling weedy coral species to proliferate within highly disturbed environments (64). Conversely, stress-tolerant corals display slower growth rates, longer life expectancies, high fecundity, and broadcast spawning strategies $(47,65)$. The larger, more robust, morphologies associated with stress-tolerant coral taxa maximise energy storage, promoting their persistence within challenging environments (66). Meanwhile, longer lifespans 
253

254

advantage of sporadic improvements in local conditions (47). Consequently, our findings support existing projections that weedy and stress-tolerant coral taxa are likely to become increasingly prevalent throughout disturbed coral communities $(67,68)$. However, these projections herald the future loss of the structural complexity considered essential to the functioning of reef ecosystems (69).

Crucially, our findings do not reflect the current reality for many coral assemblages within regions of high environmental variability, suggesting that the composition of coral communities is not solely mediated by the interplay between transient dynamics and abiotic variability. Despite the lower amplificatory capacity we report for subtropical competitive corals compared to the subtropical weedy and stress-tolerant assemblages, competitive coral taxa dominate many subtropical coral assemblages (70-72). Utilising fast growth strategies, colonies of competitive coral taxa are capable of rapidly colonising available substrate, quickly outcompeting heterospecific colonies for both space and light (47). Whilst this competitive nature perhaps explains the enhanced amplificatory capacity of the tropical competitive assemblages relative to the tropical stress-tolerant and weedy assemblages (Fig. 3A), the sensitivity of many competitive coral taxa to environmental change means that these assemblages are often regarded as early successional, dominating only within optimal environments and receding as reef ecosystems approach climax states (73, 74). Within subtropical environments, however, coral community composition is mediated by environmental pressures and dispersal barriers that filter the occurrence of species according to their trait characteristics $(46,75)$. As a result, subtropical coral assemblages typically consist of a subset of tropical species found on tropical coral reefs (46), as well as subtropical specialists and endemics. The dominance of competitive coral taxa within subtropical coral assemblages, despite their reduced transient performance relative to other coral taxa, therefore, implies that competitive interactions profoundly influence the performance of coral 
populations $(76,77)$. Certainly, further investigation into the influence of competitive interactions on the transient dynamics of coral populations is needed to disentangle how the dynamics of coexistence between coral populations facilitate their persistence within variable environments.

\section{Conclusions}

Limitations in our understanding for the abiotic determinants driving the dynamics of coral assemblages inhibits our capacity to forecast their future performance and, therefore, manage global coral community reassembly (78-80). Here, we demonstrate how coral assemblages within regions of high environmental variability exhibit demographic strategies associated with enhanced transient potential, but at a cost to their long-term performance (Fig. 2). Climatic change is exposing coral communities worldwide to increased abiotic variability. Crucially, our findings here emphasize that whilst coral assemblages can adopt demographic strategies to enhance their viability when exposed to abiotic variability, the winners and losers within future, more variable environments cannot be predicted from existing measures of long-term performance. However, the relationship that we observed between transient potential and thermal variability was not universal across coral taxa, nor did it manifest identically across hemispheres. Subtle patterns in the association between population dynamics and their climate drivers hinder predictions of the consequences of environmental change within biological communities (81). Nevertheless, relative to competitive coral taxa, weedy and stress-tolerant corals appear to possess a greater capacity for enduring within environments characterised by repeated abiotic disturbances. Yet, competitive coral taxa are often associated with more complex morphologies and therefore support the structural complexity critical to the wider functioning of coral associated ecosystems (69). Accordingly, future increases in abiotic variability threaten the viability of coral associated ecosystems. 
Methods

\section{Modelling population dynamics}

Integral Projection Models (IPMs) capture the influences of the state composition of individuals on the performance of populations over discrete time periods ( $t$ to $t+1 ; 51)$. Here, of coral populations, we used IPMs describing patterns in colony survival $(\sigma)$, transitions in size (growth and shrinkage, $\gamma$ ), fragmentation probability $(\kappa)$, fecundity $(\varphi)$, and recruitment $(\phi)$, each as a function of colony size ( $z$; visible horizontal surface area, $\left.\mathrm{cm}^{2}\right)$. Specifically, our

IPMs took the form

$$
F_{Z^{\prime} Z}=\varphi_{Z} \phi C_{0}
$$

with $[\mathrm{L}, \mathrm{U}]$ representing the range of possible colony sizes; calculated as $10 \%$ above and below observed maximum and minimum colony sizes to avoid accidental exclusion (82). Accordingly, the structure of a population at time $t+1\left(n\left[z^{\prime}, t+1\right]\right)$ is a product of its structure at time $t\left(n\left[z^{\prime}, t\right]\right)$ subject to the survival $\left(\sigma_{z}\right)$ and transition of individual colonies from size $z$ to size $z^{\prime}\left(\gamma_{z^{\prime}} z\right)$; the probability of colony fragmentation $\left(\kappa_{z}\right)$ and the number $\left(\kappa b_{z}\right)$ and size distribution of any colony remnants produced $\left(\kappa_{Z}^{0}\right)$; and colony fecundity $\left(\varphi_{z}\right)$ combined with the probability of successful recruitment $(\phi)$ and the size distribution of surviving recruits $\left(\mathrm{C}_{0}\right)$.

\section{Data Collection}

We parameterised our IPMs using data collected during repeated annual surveys of 3171 tagged colonies within tropical and subtropical coral communities in southern Japan and eastern 
colonies using permanent plots arranged haphazardly throughout four focal coral communities in situ to the lowest possible taxonomic level (either genus or species). No samples were taken from tagged colonies, as although this would have allowed us to resolve species identity, we wanted to avoid any lasting interference with the processes of colony survival, growth, and fragmentation.

To facilitate comparing population characteristics observed across spatially distinct regions in Australia and Japan with varying degrees of species overlap (83), we grouped tagged colonies across each region according to shared life-history-strategies (sensu 47-49), primarily delineated based on their morphology, growth rate and reproductive mode (47). Specifically, we categorised colonies as 'competitive', 'weedy', 'stress-tolerant' or 'generalist' following the genera classifications of Darling et al. (47), with minor adaptions made based on local expertise (see supplementary $\mathrm{S} 2$ for a detailed list). In the event that genera represented species classified across different categories (19 cases), we randomly assigned individuals across the relevant categories in proportion with the number of species within each category known to occur in the area (sensu 49). Following the pooling of colonies according to their life-historystrategies, we omitted all individuals defined as generalists from subsequent analyses due to their limited representation across our regional samples $(\mathrm{n}$ : AS $=22$ colonies; $\mathrm{AT}=31$; JS $=$ 17; JT = 65). Consequently, we constructed IPMs concerning the dynamics of 12 coral assemblages corresponding with competitive, stress-tolerant, and weedy coral taxa across four geographical locations (Fig. 1).

Photographs capturing the visible horizontal extent of tagged colonies were used to follow individuals over successive surveys and obtain longitudinal records of colony surface area $\left(\mathrm{cm}^{2}\right.$; transformed to a $\log _{10}$ scale $)$ over time. Using generalised linear mixed models 
(GLMMs), we estimated size-specific patterns in colony survival $(\sigma)$, transitions in size $(\gamma)$, autocorrelation between observations and within-subject variability associated with our pooling of data recorded from individuals followed across multiple years, and at different sites.

Colony survival $(\sigma)$ reflected the continued presence of tagged individuals across survey intervals $(t$ to $t+1)$ and was modelled as a logistic function of colony surface area at time $t$.

Colony size transitions $(\gamma)$, representing both growth through colony extension, and shrinkage through partial mortality (84), were modelled using the polynomial relationship between initial colony surface area at time $t$ and subsequent surface area at time $t+1$. Colony fragmentation probability $(\kappa)$ was then modelled as a polynomial logistic function of colony size at time $t$.

During our surveys, we recorded fragmentation in the event of observed colony breakage, recording the size (surface area, $\mathrm{cm}^{2}$ ) of all remnants produced in each case. Subsequently, we also modelled the number $\left(\kappa b_{z}\right)$ and size $\left(\kappa_{Z}^{0}\right)$ of remnant colonies produced during fragmentation as a function of colony size at time $t$, using Poisson and polynomial GLMMs, respectively.

Alongside our surveys of tagged individual colonies, we also monitored colony recruitment within our permanent coral plots. During each annual survey, we recorded the number and size of new colonies appearing within each plot. These recruitment counts enabled us to quantify annual and regional variability in recruit densities (Table S2), as well as estimate parameterising recruitment dynamics within our IPMs, we determined patterns in colony 372 fecundity $(\varphi)$. This approach was necessary because evaluating population performance requires an explicit consideration of fecundity to link the dynamics of existing individuals with the introduction of new, genetically distinct individuals (21). Using data relating colony size 
and larval output (larval density, $\mathrm{cm}^{3}$ ) extracted from the Coral Trait Database $(85,86)$, we calculated colony fecundity $(\varphi)$ as the polynomial relationship between colony size at $t$ and expected larval output (Supplementary S1). Next, to ensure our modelled recruitment dynamics accurately reflected our empirical observations we parameterised a recruit survival function $(\phi)$ within our IPMs. Here, recruit survival $(\phi)$ serves as a probability function converting expected larval output into a proportional contribution of observed recruit counts as a function of colony size, which we calculated by dividing total expected larval output in any given year by the corresponding annual recruitment count (Supplementary S1, sensu 68, 87).

\section{Quantifying population characteristics}

From our IPMs, we obtained estimates of long-term performance (asymptotic population growth, $\lambda$ ), generation time $(T)$, and transient potential (damping ratio $[\rho]$, maximal amplification $\left[\bar{\rho}_{\max }\right] \&$ transient envelope $\left.[T E]\right)$ for each tropical and subtropical coral assemblage $(21,24,52,55,88)$. Estimates of $\lambda$ are typically used as a measure of long-term population viability (22), and reflect whether a population is expected to grow $(\lambda>1)$ or decline $(\lambda<1)$ when at stationary equilibrium $(21)$. Generation time is a measure of population turnover, describing the time needed for individuals of a population to be replaced (55). Alternatively, the measures of transient potential describe the expected characteristics of populations following their displacement from stationary equilibrium due to disturbances. The damping ratio constitutes a measure of demographic recovery $(52,89)$, describing the rate at which a population perturbed from its stationary equilibrium converges back to its asymptotic growth trajectory (21). Meanwhile, maximal amplification quantifies the greatest increase in population size following a disturbance, relative to its asymptotic growth trajectory $(24,88)$. Finally, the transient envelope quantifies the magnitude by which the transient dynamics of a population deviates from its long-term trajectory (52). 

per (90). Estimates of $\lambda$ were then identified as the dominant eigenvalue of each discretised matrix, whilst we estimated damping ratios as the ratio between the subdominant and dominant eigenvalues. With the $R$ package Rage (91) we then calculated generation time using estimates of net reproductive rate $\left(R_{0}\right)$ and $\lambda$ obtained from each matrix,

$$
T=\log \left(R_{0}\right)-\log (\lambda)
$$

Next, we determined the transient envelope of each assemblage using their associated Kreiss bounds of amplification $\left(\bar{K}_{\lambda}^{*}\right)$ and attenuation $\left(\underline{K}_{\lambda}^{*}\right)$,

$$
T E=\bar{K}_{\lambda}^{*}-\underline{K}_{\lambda}^{*}
$$

Respectively, the Kreiss bounds of amplification and attenuation reflect the largest and smallest expected long-term density of a population following the dissipation of transient conditions, relative to its asymptotic growth trajectory (92-94). We acknowledge here that this definition is more commonly applied to measures of population inertia (24), which are more typically used in estimating transient envelopes (52). However, Kreiss bound estimates have been demonstrated to align with corresponding estimates of population inertia and, unlike estimates of population inertia, are not sensitive to imprimitive population models (i.e., non-negative models permitting transitions between all state classes, but with transitions between certain stages occurring only at periodic intervals; 21,24$)$; hence their selection here. We derived these Kreiss bounds, alongside estimates of maximal amplification, using their corresponding 421 functions in the $R$ package popdemo (95). 
Across each demographic measure, we determined the variance in our assemblagereplacement, whilst permitting recruit survival probabilities $(\phi)$ to vary within observed limits. Finally, prior to their inclusion in further analyses, the jack-knifed distributions of the $\lambda$, generation time, transient envelope, and maximal amplification variables required transforming to ensure approximate normality. We omitted 26 variants for which $\lambda>2$, as these presented unrealistic illustrations of population performance (i.e., more than doubling population size every year), before applying a log transformation to the generation time variable and a power transformation $\left(y^{x}\right)$ across the damping ratio $\left(y^{-2.0}\right)$, transient envelope $\left(y^{-}\right.$ $\left.{ }^{0.1}\right)$ and maximal amplification variables $\left(y^{-0.5}\right)$.

\section{Evaluating spatial trends in population characteristics}

To test for patterns in the spatial variation of long-term performance and transient potential across tropical and subtropical coral assemblages, we utilised partial least squares regression, ANOVA, and Type 2 linear regression. Initially, we applied a partial least squares regression to test whether trade-offs between the long-term performance characteristics and transient potential of coral assemblages align with their exposure to abiotic variability. Partial least squares regression quantifies the association between multiple predictor variables and one or more dependant variables (96). Subsequently, using this technique we simultaneously evaluated the relationships between mean estimates of $\lambda$, damping ratio, and transient envelope drivers. 
To evaluate how abiotic variability mediates the trade-off between the short- and longthree measures of local sea surface temperature (SST) regimes: mean monthly SST ( $\left.\overline{\mathrm{X}}_{\text {sst }}\right)$, monthly SST variance ( $\left.\mathrm{cv}_{\text {sst }}\right)$, and monthly SST frequency spectrum ( $\beta_{\text {sst }}$; Supplementary S3). Focusing on the four geographical regions in which our focal coral assemblages were surveyed (GPS: $\mathrm{AS}=-30.3^{\circ}, 153.1^{\circ} ; \mathrm{AT}=-23.4^{\circ}, 151.9^{\circ} ; \mathrm{JS}=32.8^{\circ}, 132.6^{\circ} ; \mathrm{JT}=26.5^{\circ}, 128.1^{\circ} ; \mathrm{Fig}$ grid) taken between January 1950 and December 2019, inclusive, from the HadISST dataset (97). Arranging these SST records into 69-year timeseries for each location, we then calculated the mean $\left(\overline{\mathrm{x}}_{\text {sst }}\right)$ and coefficient of variance $\left(\mathrm{cv}_{\text {sst }}\right)$ for each timeseries. Next, we estimated the frequency spectrum of each time series. Spectral analysis is used to quantify the periodicity of recurrent variability within a timeseries, with higher frequencies associated with shorter-term fluctuations (98). The frequency spectrum of a time series is represented by its spectral exponent $(\beta)$ and equal to the negative slope between its log spectral density and log frequency (99), which we calculated using the package stats (100). After testing these abiotic predictor variables for collinearity (Supplementary S3), we performed our partial least square regression analyses using the $R$ package plsdepot (101).

Next, we assessed how patterns in the long-term performance, and capacity for coral assemblages to benefit from recurrent disturbance vary between tropical and subtropical regions, and how this variation manifests across coral taxa. Using a three-way ANOVA, we separately investigated variation in estimates of $\lambda$ and maximal amplification across the three factors of country (Australia vs. Japan), ecoregion (tropical vs. subtropical), and assemblage classification (competitive, stress-tolerant or weedy). With maximal amplification estimates inverted during transformation, larger values subsequently reflect reduced amplification 
470

471

potential. For the purposes of clarity in this analysis, we will henceforth refer to this reversed scale as a demographic stability index (DSI), with lower values corresponding with enhanced amplification. Finally, we evaluated drivers of long- and short-term performance, by using Type 2 linear regression to separately evaluate the relationship between generation time $(T)$ and estimates of $\lambda$ and transient envelope (TE). Type 2 linear regression is an approach for quantifying the relationship between two non-independent variables, such that both variables include an element of error (54). Here, due to differences in the magnitude of the variance $\left(\sigma^{2}\right)$ across our variables of generation time, $\lambda$, and transient envelope $\left(\sigma^{2}: T=1.139 ; \lambda=0.009 ; T E\right.$ $=0.016)$ we performed a Ranged Major Axis regression using the $R$ package lmodel2 (102).

\section{Acknowledgements}

The authors would like to thank S. Dalton, L. Lachs, C. Kim, R. Edgar, K.-L. Gomez-Cabrera, N. Kyriacou, I. Mizukami, H. Kise, C. Fourreau, G. Masucci, P. Biondi, S. Nishihira, M. Tamae, H. Nakakoji, C. Tan, L. Lawrence, T. Hofmann, I. Montero-Serra, and all staff at Dive Quest, Pacific Marine and SeaAir for their assistance with field data collection. Additionally, aspects of this research would not have been possible without the research permits provided by the Solitary Islands Marine Park branch of the NSW Department of Primary Industries (SIMP 2016/002V2, MEAA 20/45) and the Great Barrier Reef Marine Park Authority (G19/42221.1). Funding for this research was provided by a Natural Environment Research Council (NERC) Doctoral Training Programme Scholarship and a Royal Geographical Society Ralph Brown Expedition Award (RBEA 03/19) to JC, the Australian Research Council Centre of Excellence for Coral Reef Studies (CE140100020) to JMP and others, the Australian Research Council Centre of Excellence for Environmental Decisions (CE110001014), a British Ecological Society small grant, the Winifred Violet Scott Charitable Trust, and the European Union's 
494 Horizon 2020 research and innovation programme under the Marie Skłodowska-Curie grant

495

496

497

498

499

500

501

502

503

504

505

506

507

508

509

510

511

512 agreement TRIM-DLV-747102 to MB. BS was supported by a Chancellor's Postdoctoral Research Fellowship from the University of Technology Sydney and a University of Sydney Fellowship.

\section{References}

1. T. P. Hughes, et al., Spatial and temporal patterns of mass bleaching of corals in the Anthropocene. Science 359, 80-83 (2018).

2. T. P. Hughes, et al., Global warming impairs stock-recruitment dynamics of corals. Nature 568, 387-390 (2019).

3. J. Kleypas, et al., Designing a blueprint for coral reef survival. Biological Conservation 257, 1-7 (2021).

4. E. McLeod, E. C. Shaver, M. Beger, J. Koss, G. Grimsditch, Using resilience assessments to inform the management and conservation of coral reef ecosystems. Journal of Environmental Management 277, 1-8 (2021).

5. G. Pecl, M. B. Araujo, J. D. Bell, J. Blanchard, T. C. Bonebrake, Biodiversity redistribution under climate change: Impacts on ecosystems and human well-being Publication Details. Science 355, 1-9 (2017).

6. Ø. Totland, J. Nyléhn, Assessment of the effects of environmental change on the performance and density of Bistorta vivipara: The use of multivariate analysis and experimental manipulation. Journal of Ecology 86, 989-998 (1998). 
7. E. B. Rivest, S. Comeau, C. E. Cornwall, The role of natural variability in shaping the response of coral reef organisms to climate change. Current Climate Change Reports 3, 271-281 (2017).

8. P. W. Boyd, et al., Biological responses to environmental heterogeneity under future ocean conditions. Global change biology 22, 2633-2650 (2016).

9. C. Parmesan, G. Yohe, A globally coherent fingerprint of climate change. Nature 421, $37-42(2003)$.

10. I. C. Chen, J. K. Hill, R. Ohlemüller, D. B. Roy, C. D. Thomas, Rapid range shifts of species associated with high levels of climate warming. Science 333, 1024-1026

11. S. E. Williams, L. P. Shoo, J. L. Isaac, A. A. Hoffmann, G. Langham, Towards an integrated framework for assessing the vulnerability of species to climate change.

12. D. P. Vázquez, E. Gianoli, W. F. Morris, F. Bozinovic, Ecological and evolutionary PLoS biology 6 (2008).

13. D. T. Iles, R. F. Rockwell, D. N. Koons, Shifting vital rate correlations alter predicted population responses to increasingly variable environments. American Naturalist 193, extinction risk of reef-building coral species at biogeographic scales. Nature Ecology and Evolution 5, 663-669 (2021). 
15. W. B. Foden, et al., Identifying the world's most climate change vulnerable species: A systematic trait-based assessment of all birds, amphibians and corals. PLOS ONE $\mathbf{8}$ (2013).

16. T. P. Dawson, S. T. Jackson, J. I. House, I. C. Prentice, G. M. Mace, Beyond predictions: Biodiversity conservation in a changing climate. Science 332, 53-58 (2011).

17. A. Hastings, Transients: The key to long-term ecological understanding? Trends in Ecology and Evolution 19, 39-45 (2004).

18. T. H. G. Ezard, et al., Matrix models for a changeable world: The importance of transient dynamics in population management. Journal of Applied Ecology 47, 515$523(2010)$.

19. J. Cant, R. Salguero-Gómez, M. Beger, Transient demographic approaches can drastically expand the toolbox of coral reef science. bioRxiv, 2021.04.08.438926 (2021).

20. A. Hastings, et al., Transient phenomena in ecology. Science 361, 1-9 (2018).

21. H. Caswell, Matrix population models: Construction, analysis and interpretation, 2nd Edition (Oxford University Press Inc, 2001).

22. E. E. Crone, et al., How do plant ecologists use matrix population models? Ecology Letters 14, 1-8 (2011).

23. A. Hastings, Transient dynamics and persistence of ecological systems. Ecology Letters 4, 215-220 (2001).

24. I. Stott, S. Townley, D. Hodgson, A framework for studying transient dynamics of population projection matrix models. Ecology Letters 14, 959-970 (2011). 
25. D. N. Koons, J. B. Grand, B. Zinner, R. F. Rockwell, Transient population dynamics: Relations to life history and initial population state. Ecological Modelling 185, 283297 (2005).

26. E. F. Camp, et al., The future of coral reefs subject to rapid climate change: Lessons from natural extreme environments. Frontiers in Marine Science 5, 1-21 (2018).

27. J. A. Burt, et al., Insights from extreme coral reefs in a changing world. Coral Reefs 39, 495-507 (2020).

28. M. Beger, B. Sommer, P. L. Harrison, S. D. A. Smith, J. M. Pandolfi, Conserving potential coral reef refuges at high latitudes. Diversity and Distributions 20, 1-13 (2014).

29. W. F. Precht, R. B. Aronson, Climate flickers and range shifts of reef corals. Front Ecol Environ 2, 307-314 (2004).

30. C. A. Tuckett, T. de Bettignies, J. Fromont, T. Wernberg, Expansion of corals on temperate reefs: Direct and indirect effects of marine heatwaves. Coral Reefs 36, 947956 (2017).

31. H. Yamano, K. Sugihara, K. Nomura, Rapid poleward range expansion of tropical reef corals in response to rising sea surface temperatures. Geophysical Research Letters $\mathbf{3 8}$, $1-6(2011)$

32. A. H. Baird, B. Sommer, J. S. Madin, Pole-ward range expansion of Acropora spp. along the east coast of Australia. Coral Reefs 31, 1063 (2012).

33. D. J. Booth, J. Sears, Coral expansion in Sydney and associated coral-reef fishes. Coral Reefs 37, 995 (2018). 
34. B. Sommer, M. Beger, P. L. Harrison, R. C. Babcock, J. M. Pandolfi, Differential response to abiotic stress controls species distributions at biogeographic transition zones. Ecography 41, 478-490 (2018).

35. J. L. McDonald, I. Stott, S. Townley, D. J. Hodgson, Transients drive the demographic dynamics of plant populations in variable environments. Journal of Ecology 104, 306314 (2016).

36. J. Cant, et al., Transient amplification enhances the persistence of tropicalising coral populations in marginal high latitude environments. BioRxiv, 2021.04.23.441133 (2021).

37. L. Chalmandrier, et al., Linking functional traits and demography to model speciesrich communities. Nature Communications 12, 1-9 (2021).

38. J. S. Madin, et al., A trait-based approach to advance coral reef science. Trends in Ecology and Evolution 31, 419-428 (2016).

39. C. Violle, et al., Let the concept of trait be functional! Oikos 116, 882-892 (2007).

40. J. P. Grime, S. Pierce, The evolutionary strategies that shape ecosystems (John Wiley and Sons Ltd., 2012).

41. B. J. McGill, B. J. Enquist, E. Weiher, M. Westoby, Rebuilding community ecology from functional traits. Trends in Ecology and Evolution 21, 178-185 (2006).

42. M. W. Cadotte, K. Carscadden, N. Mirotchnick, Beyond species: Functional diversity and the maintenance of ecological processes and services. Journal of Applied Ecology 48, 1079-1087 (2011). 
602

603

604

605

606

607

608

609

610

612

613

614

615

616

617

618

619

620

621

622

623

43. D. S. Falster, Å. Brännström, M. Westoby, U. Dieckmann, Multitrait successional forest dynamics enable diverse competitive coexistence. Proceedings of the National Academy of Sciences of the United States of America 114, E2719-E2728 (2017).

44. J. S. Madin, M. O. Hoogenboom, S. R. Connolly, Integrating physiological and biomechanical drivers of population growth over environmental gradients on coral reefs. Journal of Experimental Biology 215, 968-976 (2012).

45. M. Álvarez-Noriega, et al., Fecundity and the demographic strategies of coral morphologies. Ecological Society of America 97, 3485-3493 (2016).

46. B. Sommer, P. L. Harrison, M. Beger, J. M. Pandolfi, Trait-mediated environmental filtering drives assembly at biogeographic transition zones. Ecology 95, 1000-1009 (2014).

47. E. S. Darling, L. Alvarez-Filip, T. A. Oliver, T. R. Mcclanahan, I. M. Côté, Evaluating life-history strategies of reef corals from species traits. Ecology Letters 15, 1378-1386 (2012).

48. E. S. Darling, T. R. McClanahan, I. M. Côté, Life histories predict coral community disassembly under multiple stressors. Global Change Biology 19, 1930-1940 (2013).

49. J. Zinke, et al., Gradients of disturbance and environmental conditions shape coral community structure for south-eastern Indian Ocean reefs. Diversity and Distributions 24, 605-620 (2018).

50. E. S. Darling, et al., Social-environmental drivers inform strategic management of coral reefs in the Anthropocene. Nature Ecology and Evolution 3, 1341-1350 (2019).

51. M. R. Easterling, S. P. Ellner, P. M. Dixon, Size-specific sensitivity: applying a new structured population model. Ecology 81, 694-708 (2000). 
52. P. Capdevila, I. Stott, M. Beger, R. Salguero-Gómez, Towards a comparative framework of demographic resilience. Trends in Ecology \& Evolution 35, 776-786 (2020).

53. M. M. Ellis, E. E. Crone, The role of transient dynamics in stochastic population growth for nine perennial plants. Ecology 94, 1681-1686 (2013).

54. R. R. Sokal, F. J. Rohlf, Biometry: The principles and practice of statistics in biological research, 3rd Editio (W. H. Freeman \& Co, 1995).

55. J. Gaillard, et al., Generation Time: A reliable metric to measure life-history variation among mammalian populations. The American Naturalist 166, 119-123 (2005).

56. C. A. Pfister, Patterns of variance in stage-structured populations: Evolutionary predictions and ecological implications. Proceedings of the National Academy of Sciences 95, 213-218 (1998).

57. R. Lande, Risks of population extinction from demographic and environmental stochasticity and random catastrophes. American Naturalist 142, 911-927 (1993).

58. W. F. Morris, D. F. Doak, Buffering of life histories against environmental stochasticity: Accounting for a spurious correlation between the variabilities of vital rates and their contributions to fitness. American Naturalist 163, 579-590 (2004).

59. C. H. Hilde, et al., The demographic buffering hypothesis: Evidence and challenges. Trends in Ecology and Evolution 35, 523-538 (2020).

60. W. F. Morris, et al., Longevity can buffer plant and animal populations against changing climatic variability. Ecology 89, 19-25 (2008). 
647

648

649

650

651

652

653

654

655

656

657

658

659

660

661

662

663

664

665

666

667

61. M. Gamelon, et al., Influence of life-history tactics on transient dynamics: A comparative analysis across mammalian populations. American Naturalist 184, 673$683(2014)$

62. M. K. Oli, The fast-slow continuum and mammalian life-history patterns: An empirical evaluation. Basic and Applied Ecology 5, 449-463 (2004).

63. N. Knowlton, The future of coral reefs. PNAS 98, 5419-5425 (2001).

64. M. Adjeroud, et al., Recovery of coral assemblages despite acute and recurrent disturbances on a South Central Pacific reef. Scientific Reports 8 (2018).

65. C. N. Klepac, D. J. Barshis, Reduced thermal tolerance of massive coral species in a highly variable environment: Reduced heat tolerance of massive corals. Proceedings of the Royal Society B: Biological Sciences 287, 19-21 (2020).

66. R. van Woesik, A. Irikawa, R. Anzai, T. Nakamura, Effects of coral colony morphologies on mass transfer and susceptibility to thermal stress. Coral Reefs $\mathbf{3 1}$, $633-639$ (2012).

67. Y. Loya, et al., Coral bleaching: the winners and the losers. Ecology Letters 4, 122$131(2001)$.

68. J. Cant, et al., The projected degradation of subtropical coral assemblages by recurrent thermal stress. Journal of Animal Ecology 90, 233-247 (2021).

69. N. A. J. Graham, K. L. Nash, The importance of structural complexity in coral reef ecosystems. Coral Reefs 32, 315-326 (2013).

70. V. J. Harriott, P. L. Harrison, S. A. Banks, The coral communities of Lord Howe Island. Marine and Freshwater Research 46, 457-465 (1995). 
669

670

671

672

673

674

675

676

677

678

679

680

681

682

683

684
71. Y. Nozawa, M. Tokeshi, S. Nojima, Structure and dynamics of a high-latitude scleractinian coral community in Amakusa, southwestern Japan. Marine Ecology Progress Series 358, 151-160 (2008).

72. K. Sugihara, et al., Latitudinal changes in hermatypic coral communities from west Kyushu to Oki Islands in Japan. Journal of the Japanese Coral Reef Society 11, 51-67 (2009).

73. S. K. Wilson, J. P. W. Robinson, K. Chong-Seng, J. Robinson, N. A. J. Graham, Boom and bust of keystone structure on coral reefs. Coral Reefs 38, 625-635 (2019).

74. H. Ohba, K. Hashimoto, K. Shimoike, T. Shibuno, Y. Fujioka, Secondary succession of coral reef communities at Urasoko Bay, Ishigaki Island, the Ryukyus ( southern Japan ) in Proceedings of the 11th International Coral Reef Symposium, (2008), pp. $321-325$.

75. T. L. Mizerek, et al., No evidence for tropicalization of coral assemblages in a subtropical climate change hot spot. Coral Reefs 40, 1451-1461 (2021).

76. J. A. Idjadi, R. H. Karlson, Spatial arrangement of competitors influences coexistence of reef-building corals. Ecology 88, 2449-2454 (2007).

77. M. Brito-Millán, B. T. Werner, S. A. Sandin, D. E. McNamara, Influence of aggregation on benthic coral reef spatio-temporal dynamics. Royal Society Open Science 6, 1-14 (2019).

78. P. J. Edmunds, et al., Evaluating the causal basis of ecological success within the scleractinia: an integral projection model approach. Marine Biology 161, 2719-2734 (2014). 
691

692

693

694

695

696

697

698

699

700

701

702

703

704

705

706

707

708

709

710

79. P. J. Edmunds, B. Riegl, Urgent need for coral demography in a world where corals are disappearing. Marine Ecology Progress Series 635, 233-242 (2020).

80. P. J. Edmunds, Vital rates of small reef corals are associated with variation in climate. Limnology and Oceanography, 1-13 (2020).

81. T. Coulson, et al., Modeling effects of environmental change on wolf population dynamics, trait evolution, and life history. Science 334, 1275-1278 (2011).

82. J. L. Williams, T. E. X. Miller, S. P. Ellner, Avoiding unintentional eviction from integral projection models. Ecology 93, 2008-2014 (2012).

83. J. E. N. Veron, M. G. Stafford-Smith, E. Turak, L. M. DeVantier, Corals of the World (2016) (November 12, 2019).

84. J. S. Madin, A. H. Baird, M. L. Baskett, S. R. Connolly, M. A. Dornelas, Partitioning colony size variation into growth and partial mortality. Biology Letters 16, 1-5 (2020).

85. J. S. Madin, et al., The Coral Trait Database, a curated database of trait information for coral species from the global oceans. Scientific Data 3, 1-21 (2016).

86. V. R. Hall, T. P. Hughes, Reproductive strategies of modular organisms: Comparative studies of reef-building corals. Ecology 77, 950-963 (1996).

87. L. Bramanti, M. Iannelli, T. Y. Fan, P. J. Edmunds, Using demographic models to project the effects of climate change on scleractinian corals: Pocillopora damicornis as a case study. Coral Reefs 34, 505-515 (2015).

88. I. Stott, M. Franco, D. Carslake, S. Townley, D. Hodgson, Boom or bust? A comparative analysis of transient population dynamics in plants. Journal of Ecology 98, 302-311 (2010). 
89. D. Hodgson, J. L. McDonald, D. J. Hosken, What do you mean, "resilient"? Trends in Ecology and Evolution 30, 503-506 (2015).

90. P. A. Zuidema, E. Jongejans, P. D. Chien, H. J. During, F. Schieving, Integral projection models for trees: A new parameterization method and a validation of model output. Journal of Ecology 98, 345-355 (2010).

91. O. R. Jones, et al., Rcompadre and Rage - two R packages to facilitate the use of the COMPADRE and COMADRE databases and calculation of life history traits from matrix population models. bioRxiv, 2021.04.26.441330 (2021).

92. S. Townley, D. Carslake, O. Kellie-Smith, D. Mccarthy, D. Hodgson, Predicting transient amplification in perturbed ecological systems. Journal of Applied Ecology $\mathbf{4 4}$ (2007).

93. S. Townley, D. J. Hodgson, Erratum et addendum: Transient amplification and attenuation in stage-structured population dynamics. Journal of Applied Ecology 45, 1836-1839 (2008).

94. H. O. Kreiss, Über Die Stabilitätsdefinition Für Differenzengleichungen Die Partielle Differentialgleichungen Approximieren. BIT 2, 153-181 (1962).

95. I. Stott, D. J. Hodgson, S. Townley, Popdemo: An R package for population demography using projection matrix analysis. Methods in Ecology and Evolution 3, 797-802 (2012).

96. L. M. Carrascal, I. Galván, O. Gordo, Partial least squares regression as an alternative to current regression methods used in ecology. Oikos 118, 681-690 (2009). 
97. N. A. Rayner, et al., Global analyses of sea surface temperature, sea ice, and night marine air temperature since the late nineteenth century. Journal of Geophysical Research 108, 1-37 (2003).

98. J. V. Greenman, T. G. Benton, The frequency spectrum of structured discrete time population models : its properties and their ecological implications. Oikos 110, 369389 (2005).

99. D. A. Vasseur, P. Yodzis, The color of environmental noise. Ecology 85, 1146-1152 (2004).

100. R Core Team, R: A language and environment for statistical computing. (2019).

101. G. Sanchez, plsdepot: Partial least squares (PLS) data analysis methods (2012).

102. P. Legendre, lmodel2: Model II regression (2018).

\section{Tables and Figures}

\section{Figure 1. Using repeated annual surveys of tagged individual colonies, conducted between} 2016 and 2019, we quantified the influence of environmental variability on the long-term performance and transient potential of tropical and subtropical coral populations in southern Japan and eastern Australia. (A) As climate shifts induce range expansions in many coral species worldwide, their populations are increasingly exposed to a gradient in thermal regimes, illustrated here by mean monthly sea surface temperatures $\left(\overline{\mathrm{x}}_{\mathrm{sst}} ;{ }^{\circ} \mathrm{C}\right)$ recorded between 1950 and 2019 (97). (B) To explore spatial patterns in the long-term performance and transient (short-term) potential of coral populations exposed to varying thermal regimes, we constructed Integral Projection Models (IPMs) describing the dynamics of tropical and subtropical assemblages of competitive, stress-tolerant, and weedy coral taxa. To parameterise 
these models, between 2016 and 2019 we documented the survival, growth, fragmentation, and recruitment patterns of 3171 tagged individual colonies within the tropical reef communities ( $\boldsymbol{\Delta})$ of Okinawa (Japan) and Heron Island (Australia), and within the subtropical coral communities $(\bullet)$ of Kochi (Japan) and the Solitary Islands Marine Park (Australia).

\section{Figure 2. A trade-off exists between long-term performance and transient potential across} our examined coral populations, corresponding with their relative exposure to thermal variability. Partial least squares regression score plot illustrating the association between thermal conditions, and the long-term performance $(\lambda)$ and transient potential (transient envelope $[T E]$ \& damping ratio $[\rho])$ of tropical $(\boldsymbol{\Delta})$ and subtropical $(\bullet)$ populations of competitive (blue), stress-tolerant (yellow), and weedy (red) coral taxa. To quantify the thermal conditions experienced by each coral population, we used sea surface temperatures (SST) recorded between 1950 and 2019 to calculate regional estimates of mean monthly SST $\left(\bar{x}_{s s t}\right)$, monthly SST variance $\left(c v_{s s t}\right)$, and monthly SST frequency spectrum $\left(\beta_{s s t}\right)$. Component scores illustrate the relative degree of variance explained in the thermal predictor variables, whilst $r_{[y]}^{2}$ reflects the cumulative variance explained across the demographic characteristics. The shaded polygons reflect the clustering of tropical and subtropical populations, whilst the dotted lines delineate regions of association to facilitate the visualisation of patterns in correlation between the abiotic and demographic variables.

Figure 3. Inter-specific variation within the trade-off observed between long-term performance and transient potential across tropical and subtropical correlates with patterns in population turnover rate (A) Interaction plot showcasing how estimates of demographic stability index (DSI) vary between associated tropical $(\mathbf{\Delta})$ and subtropical $(\bullet)$ populations of competitive (blue), stress-tolerant (yellow), and weedy (red) coral taxa in 
781 Australia and Japan. We present DSI, as an inverse measure of maximal amplification $\left(\bar{\rho}_{\max }\right)$,

782 describing the ability for populations to undergo elevated growth following disturbance. Thus,

783 lower DSI estimates correspond with enhanced amplification capacity. We also applied Type

7842 linear regression to separately explore the association of population turnover characteristics

with (B) long-term performance (asymptotic population growth rate; $\lambda$ ), and (C) transient potential (transient envelope, $T E$ ) across tropical and subtropical populations of competitive, stress-tolerant, and weedy coral taxa in Australia and Japan. We note here that transient envelope estimates were reversed during transformation to achieve normality, thus higher values reflected diminished transient potential. We have therefore displayed transient potential on a reversed scale to facilitate comparisons with patterns in long-term performance $(\lambda)$. We used generation time (displayed here on the log scale) as a measure of population turnover rate, 
A.

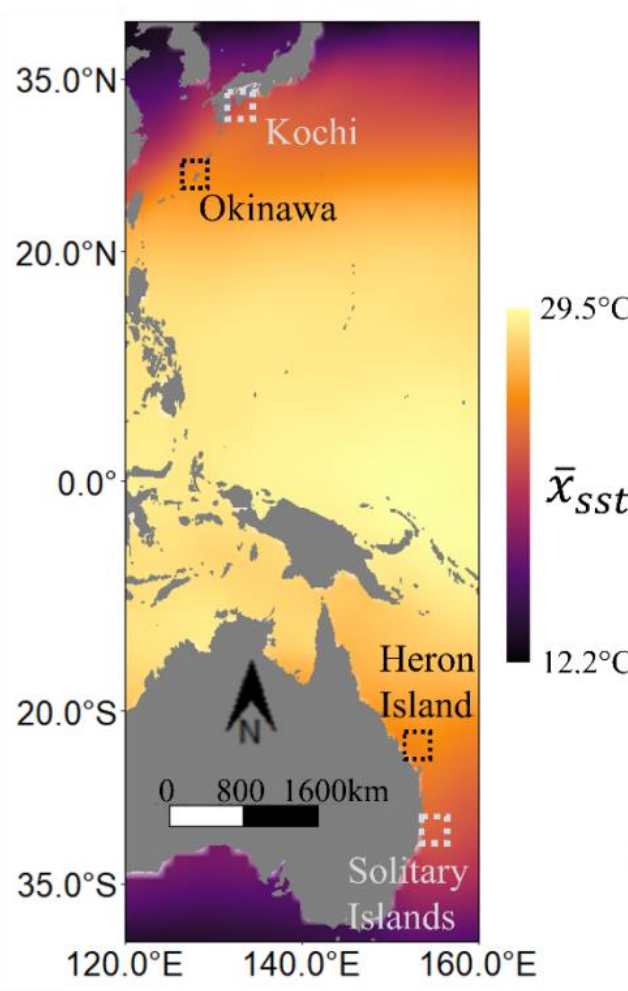

B.

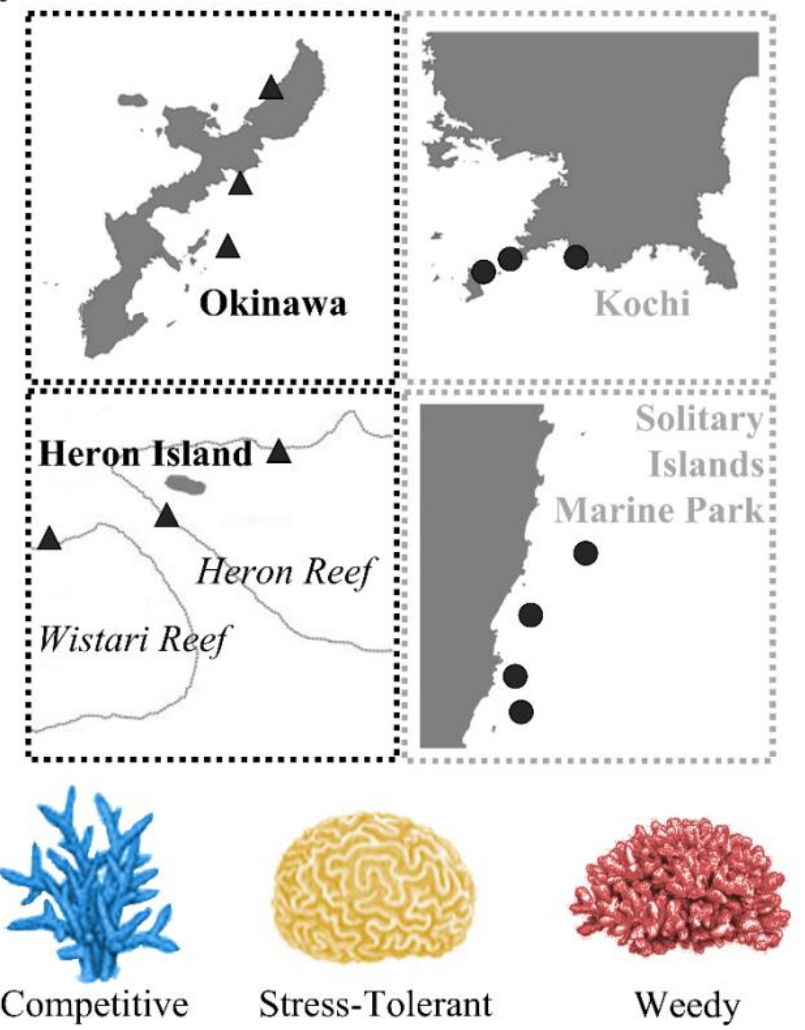

804

805

806

807

808

809

810

811 
bioRxiv preprint doi: https://doi.org/10.1101/2021.09.29.462350; this version posted October 1,2021 . The copyright holder for this preprint

(which was not certified by peer review) is the author/funder, who has granted bioRxiv a license to display the preprint in perpetuity. It is made available under aCC-BY-NC-ND 4.0 International license.

Page 36 of 38

\section{$813 \quad$ Figure 2}

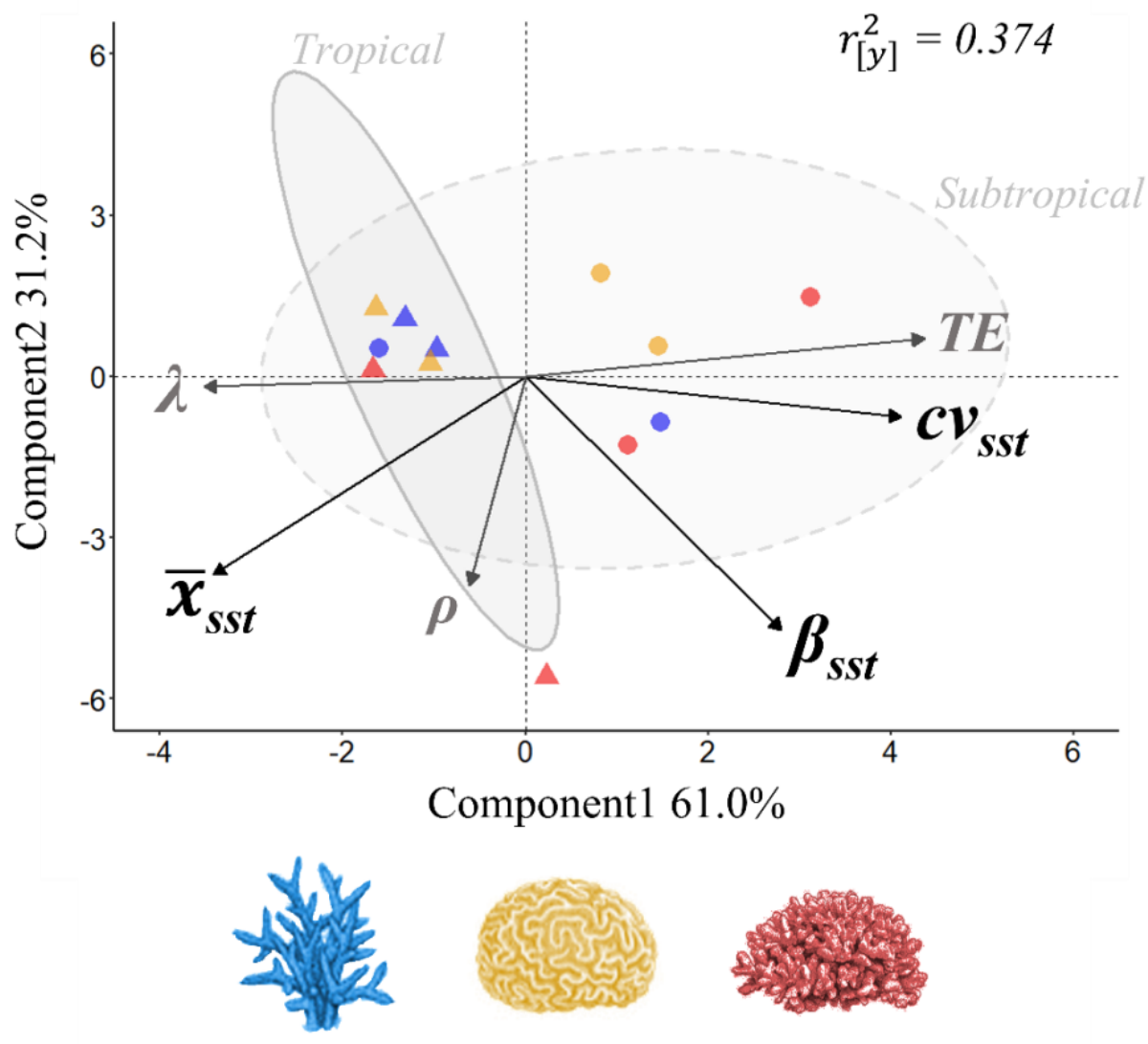

814

815

816

817

818

819

820

821

822 
bioRxiv preprint doi: https://doi.org/10.1101/2021.09.29.462350; this version posted October 1,2021 . The copyright holder for this preprint

(which was not certified by peer review) is the author/funder, who has granted bioRxiv a license to display the preprint in perpetuity. It is made available under aCC-BY-NC-ND 4.0 International license.

Page 37 of 38

Figure 3

A.

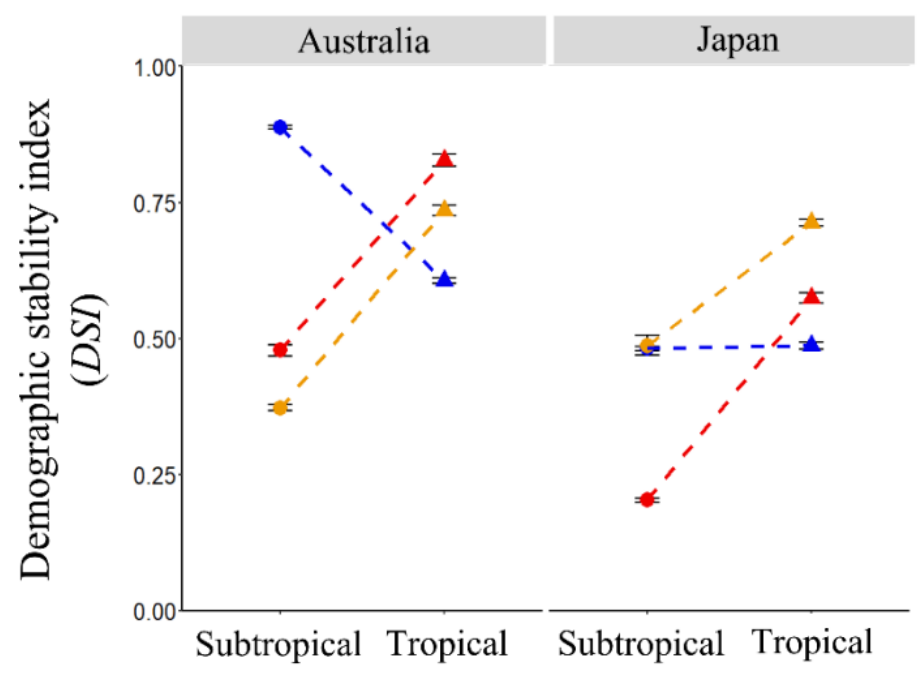

B.

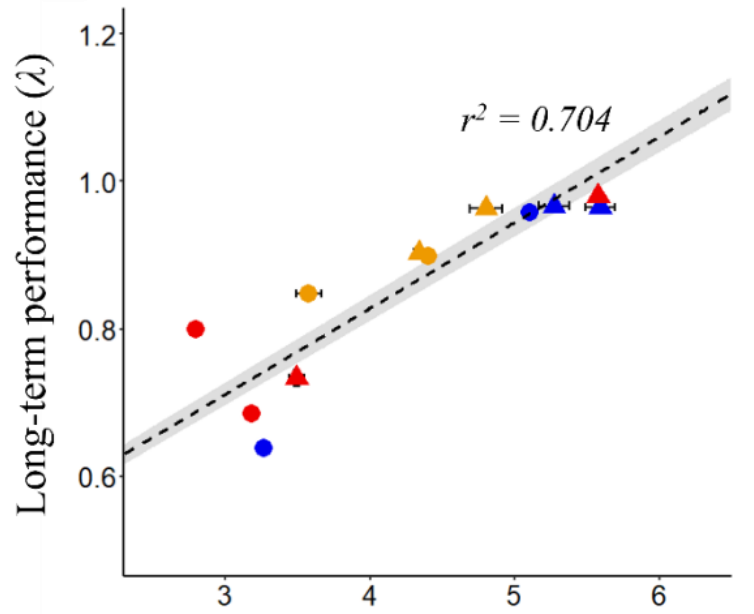

Generation time ( $T$ )
C.

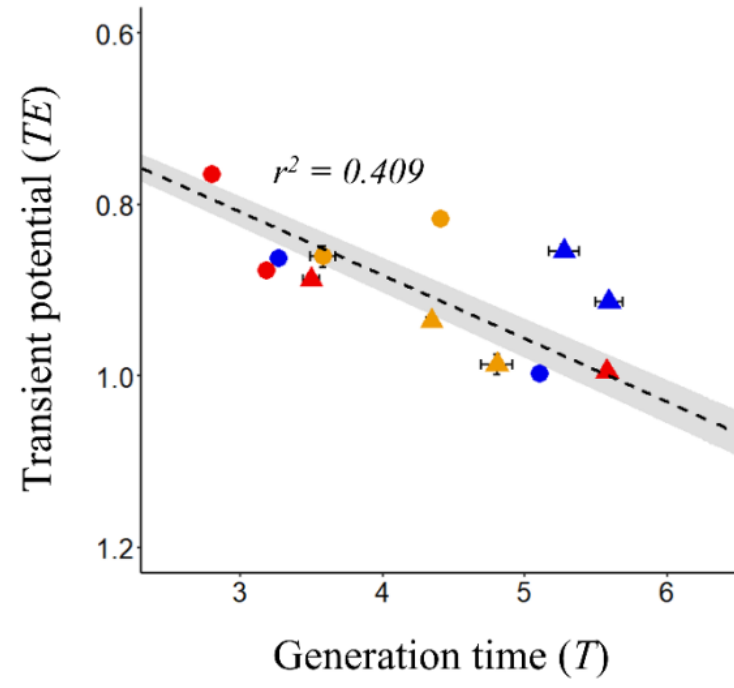

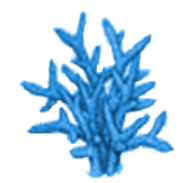

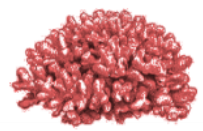


830 Table 1. Population growth rates $(\lambda)$ obtained from corresponding tropical and subtropical

831 populations of competitive, stress-tolerant, and weedy coral taxa in Australia and Japan.

832 Shading used to highlight the highest estimate of population growth across each tropical-

833 subtropical pairing. Error displayed using 95\% CI.

\begin{tabular}{c|ccc} 
Country & Life-history group & Tropical & Subtropical \\
\hline \multirow{3}{*}{ Australia } & Competitive & $0.983[0.981,0.984]$ & $0.958[0.957,0.959]$ \\
& Stress-tolerant & $0.983[0.980,0.985]$ & $0.899[0.898,0.899]$ \\
& Weedy & $0.981[0.980,0.982]$ & $0.686[0.684,0.687]$ \\
\cline { 2 - 4 } Japan & Competitive & $1.001[0.999,1.004]$ & $0.640[0.639,0.641]$ \\
& Stress-tolerant & $0.913[0.909,0.917]$ & $0.885[0.877,0.894]$ \\
& Weedy & $0.760[0.750,0.770]$ & $0.807[0.802,0.812]$
\end{tabular}

